regions as hydroxychloroquine was more frequently used in LA and injectable gold and sulfasalazine were more frequently used in EUR. The new DMARDs were being used more in the US, probably due to earlier regulatory agency approval.

\section{SAT0256 ECONOMIC IMPACT OF INFLIXIMAB TREATMENT IN RHEUMATOID ARTHRITIS; CORRELATION WITH DISEASE ACTIVITY SCORE}

${ }^{1} \mathrm{~A}$ Kavanaugh, ${ }^{2} \mathrm{~K}$ Patel, ${ }^{2} \mathrm{M}$ Bala, ${ }^{3} \mathrm{~L}$ Noe, ${ }^{4} \mathrm{C}$ Antoni. ${ }^{1}$ Rheumatology, University of California at San Diego, La Jolla, USA; ${ }^{2}$ Biostatistics, Centocor, Inc., Malvern; ${ }^{3}$ Ovation Research Group, Highland Park, USA; ${ }^{4}$ Medicine, University Erlangen-Nuremberg, Erlangen, Germany

\subsection{6/annrheumdis-2001.892}

Background In the ATTRACT trial, rheumatoid arthritis (RA) patients treated with the anti-TNF monoclonal antibody infliximab showed significant improvement in the signs and symptoms of disease (e.g. as measured with using the Disease Activity Score [DAS]); treatment also achieved improvement in quality of life measures and inhibited the progression of structural damage at 54 weeks. The ultimate clinical utility of biologic agents may be impacted by their high acquisition costs. However, RA is itself associated with substantial direct and indirect costs that vary with the severity of disease. Therefore, highly effective therapies for RA may be cost effective.

Objectives To assess the total costs of treating RA, exclusive of the costs of study medication, for patients enrolled in the ATTRACT trial, and to correlate total costs with disease activity and response to therapy using the DAS.

Methods Economic evaluation was performed on patients in the ATTRACT trial over 54 weeks of study. The economic impact of improvement in disease activity was assessed by analysing costs according to clinical response using the DAS. DAS28 was calculated as follows:

$0.56 * \mathrm{SQRT}(\mathrm{TJC})+0.28 * \mathrm{SQRT}(\mathrm{SJC})+0.7 \ln$ (ESR) + $0.014 *$ (pt global (VAS)).

Clinical responses in the trial were characterised as 'None', 'Moderate' or 'Good' using DAS scores at endpoint and change in DAS during the study. Categorical variables were tested using Cochran-Mantel-Haenszel test.

Results Clinical efficacy at 54 weeks was significantly greater among patients receiving infliximab compared to those receiving placebo $(\mathrm{p}=0.001)$. Total cost among all patients with ?Good? DAS response was $\$ 3,836$ compared to $\$ 5,613$ and $\$ 13,125$ among patients with ?Moderate? and ?None? response respectively. Thus, patients who had a ?Good? DAS response cost $\$ 9,289$ less on average, compared to ?None? DAS response. DAS28 response rates and total cost by DAS response are presented below.

\begin{tabular}{llll}
\multicolumn{4}{l}{ Abstract SAT0256 Table 1 } \\
\hline & Good & Moderate & None \\
\hline Pts. evaluated & 110 & 112 & 205 \\
Placebo DAS28 Response & $5.7 \%$ & $15.9 \%$ & $78.4 \%$ \\
Infliximab DAS28 Response & $30.9 \%$ & $28.9 \%$ & $40.2 \%$ \\
Mean \pm SD & $\$ 3,836 \pm \$ 2,893$ & $\$ 5,613 \pm \$ 6,640$ & $\$ 13,125 \pm \$ 29,119$ \\
Median & $\$ 2,965$ & $\$ 3,386$ & $\$ 5,530$ \\
Interquartile range & $(\$ 1,960, \$ 4,856)$ & $(\$ 2,170, \$ 6,130)$ & $(\$ 2,889, \$ 10,501)$ \\
Range & $(\$ 433, \$ 16,717)$ & $(\$ 141, \$ 39,610)$ & $(\$ 226, \$ 350,138)$ \\
\hline
\end{tabular}

Conclusion Improvement in DAS significantly reduced the cost of treating RA patients. In patients with severe RA, therapy with infliximab was effective, as assessed by DAS. Moreover, patients achieving moderate and good DAS responses incurred significantly less costs over 54 weeks of therapy than non-responders. This data shows that among patients with severe RA, highly effective therapy, such as infliximab, can reduce costs. Such therapy may be cost effective.

\section{SAT0257 SOME DATA ABOUT THE HOSPITAL CARE AND DISABILITY RATE OF RA IN HUNGARY}

${ }^{1} \mathrm{P}$ Géher, ${ }^{2} \mathrm{G}$ Héjj, ${ }^{3} \mathrm{~L}$ Kincses. ' Chair of Rheumatology and Physiotherapy, Semmelweis University; ${ }^{2}$ Department of Rheumatology, National Institute of Rheumatology and Physiotherapy, Budapest; ${ }^{3}$ Direction, Center for Healthcare Information of the Ministry of Health, Szekszárd, Hungary

\subsection{6/annrheumdis-2001.893}

Background Based upon population studies the prevalence of RA in Hungary is $1.01 \%$ among the adult population. (The estimated number of RA cases is 84,455 .) RA is characterised by early disability and frequent hospitalisation. Health related quality of life of RA patients can be improved significantly with joint surgery.

Objectives To describe the annual number of hospitalisation due to RA, the annual number of large joint surgery performed among RA patients, the number of new case of RA with permanent disability.

Methods From the national hospital database all cases with the main diagnosis of RA (ICD code group M05 and M06) were selected for analysis. Multiple hospitalisation of the same patients were excluded. The number of disability pension for RA was drawn from the annual report of the National Institute for Medical Experts.

Results There were 2,556,338 hospitalisation in Hungary in 1999. In 5,243 cases (0.2\%) RA was the main diagnosis. This represents the $6.2 \%$ of all RA patients, calculated on the epidemiological data. In this year 7,106 total hip replacement and 2,539 knee replacement were performed, among them there were 75 cases of hip, and 78 cases of knee surgery in RA. Reconstructive surgery represents $2,9 \%$ of the total hospitalisation with RA. There were 3,667 new disability claimants with locomotor diseases (with any of the "M" ICD code) which is $10.6 \%$ of the total new pensionist. RA was the leading cause of disability in 231 (6.2\%) cases.

Conclusion Our hospitalisation data with RA are conflicting with the estimated total number of RA patients. Surgery (as measured by the number of large joint reconstuctive surgery) represents only a small proportion as an indication for hospitalisation with RA. 